\section{Frequência e características maternas e do recém nascido associadas à internação de neonatos em UTI no município de Joinville, Santa Catarina - 2012}

\section{The frequency and characteristics of mothers and newborns associated with the admission of neonates to ICU in the municipality of Joinville, Santa Catarina - 2012}

Fátima Mucha 1

Selma Cristina Franco 2

Guilherme Alberto Germano Silva 3

\begin{abstract}
Objectives: to determine the frequency of and factors associated with the admission of newborns to neonatal ICU in the municipality of Joinville, Santa Catarina.

Methods: a cross-sectional study was conducted based on the records of live births (DNV) and admissions to neonatal intensive care units (NICUs) in 2012. To evaluate associations between the explanatory variables and the outcome, the crude and adjusted odds ratios (OR) were calculated with respective confidence intervals of $95 \%$ using the logistic regression technique.

Results: the frequency of admission to NICU was $11.7 \%$ (9.69-13.7). Low birth weight (ORadj $=8.1$ $[6.3-10.3])$, congenital deformity (ORadj $=6.0[3.2$ - 11.2]), a 5th minute Apgar score $<7$ (ORadj $=5.7$ $[2.6-12.3])$, prematurity (ORadj $=3.4[(2.7-4.1])$, male sex (ORaj $=1.5$ [1.2 - 1.8]), public hospital $($ ORadj $=2.0[1 ; 6-2.4])$, single mother (ORadj $=$ $1.4[1.2-1.7])$, low level of education of mother $($ ORadj $=1.4[1.2-1.7])$ and $<7$ prenatal consults $($ ORadj $=1.3[1.1-1.6])$ were found to be risk factors for admission.

Conclusions: the biological characteristics of the newborns associated with admission to neonatal intensive care units are susceptible to prevention, thereby demonstrating the importance of improving care for pregnant women and newborns.
\end{abstract}

Key words Risk factors, Intensive care units, neonatal, Maternal and child health
Resumo

Objetivos: determinar a frequência e os fatores associados à internação de recém-nascidos em UTI neonatal no municipio de Joinville, Santa Catarina.

Métodos: estudo transversal com base nos registros de nascidos vivos (DNV) e de internações em Unidades de Terapia Intensivas (UTI) neonatais em 2012. Para avaliar associações entre variáveis explicativas e desfecho foram estimados odds ratios (OR) brutos e ajustados e respectivos intervalos de confiança de $95 \%$ com a técnica de regressão logística.

Resultados: a frequência de internação em UTI neonatal foi de 11,7\% (9,69-13,7). Baixo peso ao nascer (ORaj $=8,1$ [6,3 - 10,3]), malformação congênita (ORaj $=6,0[3,2-11,2])$, Apgar de $5^{\circ}$ minuto $<7($ ORaj $=5,7[2,6-12,3])$, prematuridade $($ ORaj $=3,4[(2,7-4,1])$, sexo masculino (ORaj $=1,5$ $[1,2-1,8])$, hospital público (ORaj $=2,0[1,6-2,4])$, mãe sem companheiro (ORaj $=1,4[1,2-1,7])$, baixa escolaridade materna (ORaj $=1,4[1,2-1,7]) e<7$ consultas no pré natal (ORaj $=1,3[1,1-1,6])$ constituíram fatores de risco para internação.

Conclusões: as características biológicas dos recém-nascidos associadas à internação em UTI neonatal são passivveis de prevenção, evidenciando a importância da qualificação da assistência à gestante e recém-nascido.

Palavras-chave Fatores de risco, Unidades de terapia intensiva neonatal, Saúde materno-infantil 


\section{Introdução}

Nos últimos anos, o avanço das tecnologias e a qualificação da assistência nas Unidades de Terapia Intensivas (UTI) neonatais vêm sendo considerados os principais responsáveis pela redução da mortalidade neonatal, especialmente nos países desenvolvidos. ${ }^{1}$ No Brasil, a redução deste componente da mortalidade infantil tem sido menor do que a verificada no componente pós-neonatal, sendo possível observar-se sua predominância. Consequentemente, a assistência neonatal ganha cada vez mais relevância e constitui um desafio a ser enfrentado.2-4

Diversos estudos descrevem o perfil dos recémnascidos internados em UTIs neonatais e identificam fatores de risco para desfechos, tais como óbito, infecção neonatal e atraso no desenvolvimento neurológico. Enquanto alguns deles evidenciam as práticas assistenciais na gestação e parto5-7 e nas UTIs, ${ }^{1}$ outros enfatizam as características biológicas maternas, tais como idade, obesidade, hipertensão e infecções $5,8-10$ ou ainda as características dos recémnascidos, especialmente a prematuridade, baixo peso ao nascer, presença de malformação congênita, Apgar de quinto minuto menor que sete 4,5,7,11-17 como determinantes da morbimortalidade dos recém- nascidos internados.

Com relação à internação em UTIs neonatais, estudos mostram a iniquidade no acesso de certos grupos populacionais $18 \mathrm{e}$ identificam fatores associados à internação tais como rotura precoce das membranas, corioamnionite, Apgar baixo no quinto minuto, mães adolescentes (idade $<20$ anos), prematuridade e baixo peso. $9,10,12,19,20$

O levantamento de literatura realizado nas bases de dados Medline, Lilacs e SciELO nos últimos cinco anos não encontrou estudos brasileiros de base populacional que quantificassem e analisassem fatores de risco para internação em UTI neonatal.

Diante disso, considerando a importância de se conhecer os fatores de risco para internação nas UTIs neonatais, os quais poderiam potencialmente contribuir para o planejamento de ações de prevenção e de aprimoramento da assistência à população materno-infantil, o presente estudo objetivou determinar a frequência e os fatores associados à internação de recém-nascidos vivos em UTI neonatal no município de Joinville, Santa Catarina.

\section{Métodos}

Estudo observacional transversal, de base populacional, realizado a partir dos nascidos vivos de mulheres residentes em município no sul do Brasil no ano de 2012.

O município de Joinville é considerado o terceiro polo econômico do sul do país, apresenta elevado índice de desenvolvimento humano $(\mathrm{IDH}=0,857) \mathrm{e}$ em 2012 apresentava uma população de $526 \mathrm{mil}$ habitantes. Em 2012, a rede básica de saúde era composta por 54 unidades e a assistência ao parto realizada em quatro hospitais, dois deles com atendimento exclusivamente pelo Sistema Único de Saúde (SUS) e responsáveis por cerca de $60 \%$ dos nascimentos de mães munícipes, e dois privados. Quase a totalidade dos partos realizados em 2012 (99,8\%) foram hospitalares. Todos os hospitais possuíam UTI neonatal totalizando 32 leitos, 17 deles nos hospitais públicos.

Os dados maternos e dos nascidos vivos foram obtidos das Declarações de Nascidos Vivos (DNV) do Sistema de Informações sobre Nascidos Vivos (SINASC) (base municipal) e compreenderam as variáveis relativas às características sociodemográficas maternas (idade, situação conjugal, escolaridade), da assistência pré-natal (mês de início do prénatal, número de consultas), ao parto (tipo de estabelecimento, tipo de parto) e às características do recém-nascido (sexo, peso ao nascer, presença de malformação, Apgar no quinto minuto de vida e idade gestacional).

O desfecho estudado foi a internação em UTI neonatal por ocasião do nascimento, de todos os nascidos vivos de mães munícipes que tiveram seu parto no próprio município em hospitais públicos ou privados, no ano de 2012. Essa informação foi obtida a partir da consulta aos relatórios dos hospitais públicos e privados que continham os dados de dos nascidos vivos internados em UTIs neonatais no período do estudo, os quais foram relacionados manualmente ao banco de dados de nascidos vivos com base nas seguintes variáveis: data do nascimento, nome da mãe e hospital de nascimento.

Os dados coletados foram revisados por dois profissionais participantes da pesquisa e armazenados em uma planilha MsExcel 2007. Inicialmente foi realizada análise descritiva das distribuições de frequências absoluta e relativa das principais características da amostra. A magnitude do efeito das variáveis explicativas foi estimada pelo cálculo do odds ratio (OR) bruto e seus respectivos intervalos de confiança de 95\% (IC95\%). As variáveis que apresentaram associação estatística com nível de significância $<0,20$ foram incluídas no modelo de regressão logística múltipla não condicional para a obtenção dos odds ratios ajustados. A seleção das variáveis no modelo final foi feita pelo método stepwise backward. Considerou-se o nível 
de significância estatística de $p<0,05$. O programa SPSS 21.0 foi utilizado nas análises.

O estudo foi aprovado pelo Comitê de Ética da Universidade da Região de Joinville (UNIVILLE), Processo $\mathrm{n}^{\mathrm{o}}$ 14836113.7.0000.5366 e por se tratar de base de dados secundária contendo dados epidemiológicos não identificados, não foi utilizado o termo de consentimento informado.

\section{Resultados}

Do total dos 7887 nascidos vivos registrados no sistema no ano de 2012, 921 necessitaram de internação em UTI neonatal, observando-se uma frequência de 11,7\% (IC95\% = 9,6 - 13,7).

$\mathrm{Na}$ Tabela 1 são apresentadas as distribuições de frequência das características maternas e as relativas ao parto, os valores dos odds ratios brutos e ajustados e os respectivos intervalos de confiança de $95 \%$ da associação desses fatores com a internação em UTI neonatal. Idade materna $<20$ anos, ausência de companheiro, baixa escolaridade materna, número de consultas no pré-natal $<7$ e nascimento em hospital público, apresentaram associação estatisticamente significante com a internação em UTI. Após ajuste, todas as variáveis se mantiveram associadas à internação em UTI, com exceção da idade materna.

\section{Tabela 1}

Distribuição de frequência e odds ratio bruto e ajustado da associação entre características sociodemográficas maternas e do parto e internação de recém-nascidos em unidade de terapia intensiva neonatal. Joinville, SC, 2012.

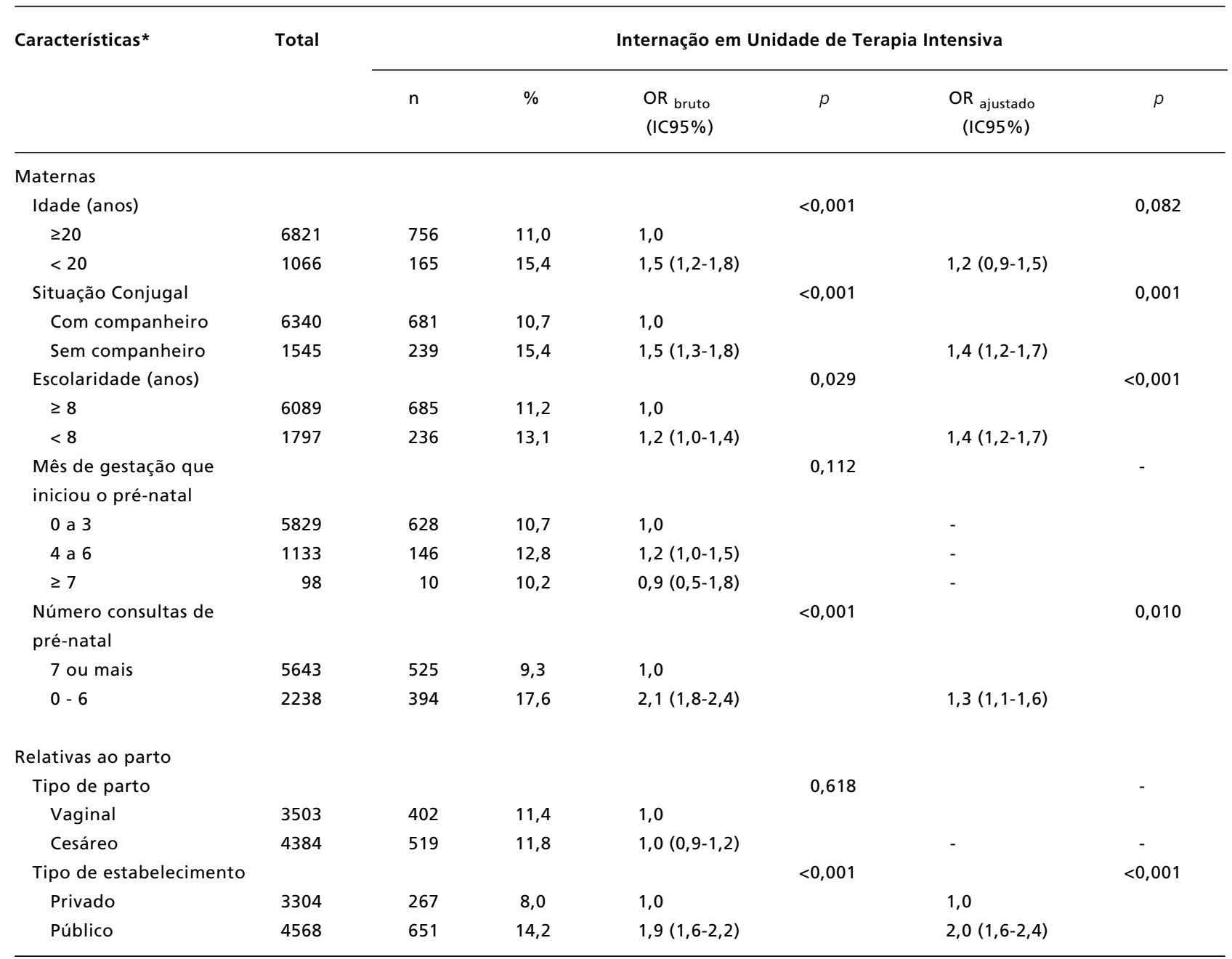

* a variação do número de nascidos vivos em cada variável deveu-se à falta de registro na DNV ou na planilha das Unidades de Terapia Intensiva.

Fonte: SINASC, Hospitais de Joinville, Janeiro a dezembro, 2012: Joinville, Brasil (2012). 
$\mathrm{Na}$ Tabela 2, são apresentadas as distribuições de frequência das características dos recém-nascidos, os valores dos odds ratios brutos e ajustados e os respectivos intervalos de confiança de $95 \%$ da associação desses fatores com a internação em UTI neonatal. Baixo peso ao nascer, malformação congênita, Apgar no quinto minuto $<7$ e prematuridade apresentaram associação estatisticamente significante com a internação em UTI. Após ajuste, todas as variáveis independentes permaneceram associadas à internação em UTI.

Observou-se associações muito fortes, com o peso ao nascer, Apgar no quinto minuto menor que 7, anomalias congênitas e prematuridade e forte, com número de consultas de pré-natal, que diminuíram a força de associação na análise multivariada, revelando estrutura de confundimento. Notou-se que essa diminuição em relação à presença de malformação congênita foi muito discreta e que a variável 'tipo de estabelecimento' manteve a magnitude da associação. A variável 'mãe adolescente' perdeu significância após ajuste do modelo e a variável 'número de consultas de pré-natal' apresentou associação limítrofe $\left(\mathrm{OR}_{\mathrm{aj}}=1,3[(1,1-1,6)]\right.$. Apesar disso, vale destacar que no modelo multivariado a força da associação de ambas as variáveis não foi desprezível.

\section{Discussão}

O presente estudo mostrou a elevada frequência de internação em UTI neonatal (em torno de 12\%) na população de recém-nascidos vivos estudada, representando uma sobrecarga considerável aos serviços de saúde pública e privada na localidade estudada. Por se tratar de um estudo de base populacional, este estudo fornece informações relevantes para o dimensionamento da infraestrutura necessária ao atendimento da demanda de saúde desta população específica na região, garantindo assim o acesso às unidades de alta complexidade.

A principal limitação deste estudo se refere à utilização de uma base de dados secundária, cuja qualidade pode ser afetada pela falta de padronização dos registros. Entretanto, é importante ressaltar que a utilização do SINASC como fonte de informação e sua integração com bases de dados hospitalares que registram o perfil dos recémnascidos internados nas UTIs neonatais constitui uma ferramenta valiosa para ser usada em saúde pública e apresenta elevada confiabilidade quando os registros são revisados e corrigidos nas bases de

Tabela 2

Distribuição de frequência e odds ratio bruto e ajustado da associação entre características do recém-nascido e internação em unidade de terapia intensiva neonatal. Joinville, SC, 2012.

\begin{tabular}{|c|c|c|c|c|c|c|c|}
\hline \multirow[t]{2}{*}{ Características* } & \multirow[t]{2}{*}{ Total } & \multicolumn{6}{|c|}{ Internação em Unidade de Terapia Intensiva } \\
\hline & & $\mathrm{n}$ & $\%$ & $\begin{array}{l}\text { OR bruto } \\
\text { (IC95\%) }\end{array}$ & $p$ & $\begin{array}{c}\mathrm{OR}_{\text {ajustado }} \\
\text { (IC95\%) }\end{array}$ & $p$ \\
\hline Sexo & & & & & $<0,001$ & & $<0,001$ \\
\hline Masculino & 3999 & 519 & 12,9 & $1,3(1,1-1,5)$ & & $1,5(1,3-1,8)$ & \\
\hline Feminino & 3888 & 402 & 10,3 & 1,0 & & & \\
\hline Peso (g) & & & & & $<0,001$ & & $<0,001$ \\
\hline$<2500$ & 604 & 339 & 56,1 & $14,7(12,9-17,7)$ & & $8,1(6,3-10,3)$ & \\
\hline$\geq 2500$ & 7283 & 582 & 7,9 & 1,0 & & & \\
\hline Malformação congênita & & & & & $<0,001$ & & $<0,001$ \\
\hline Não & 7820 & 891 & 11,3 & 1,0 & & & \\
\hline Sim & 67 & 30 & 44,7 & $6,3(3,9-10,3)$ & & $6,0(3,2-11,2)$ & \\
\hline Apgar $5^{\circ}$ minuto & & & & & $<0,001$ & & $<0,001$ \\
\hline$\geq 7$ & 7807 & 878 & 11,2 & 1,0 & & 1,0 & \\
\hline$<7$ & 72 & 42 & 58,3 & $11,1(6,9-17,7)$ & & $5,7(2,6-12,3)$ & \\
\hline Prematuridade & & & & & $<0,001$ & & $<0,001$ \\
\hline Não & 6908 & 538 & 7,7 & 1,00 & & & \\
\hline Sim & 979 & 383 & 39,1 & $7,6(6,5-8,9)$ & & $3,4(2,7-4,1)$ & \\
\hline
\end{tabular}

* a variação do número de nascidos vivos em cada variável deveu-se à falta de registro na DNV ou na planilha das Unidades de Terapia Intensiva.

Fonte: SINASC, Hospitais de Joinville, Janeiro a dezembro, 2012: Joinville, Brasil (2012). 
dados. ${ }^{21}$ Outra limitação refere-se ao delineamento transversal do estudo que não permite realizar inferências causais em relação ao desfecho estudado.

A ocorrência de internação em UTI neonatal na população estudada foi semelhante à relatada em um estudo de abrangência nacional conduzido no Canadá entre 2006 e 2009, cujo percentual de internações dos recém-nascidos em UTI foi de $11,1 \%$ dos nascidos vivos. ${ }^{22}$ Considerando a diferença das duas realidades, Joinville e Canadá, não fica clara uma explicação para a semelhança dos índices observados. Como o índice canadense é uma média nacional, as regiões menos desenvolvidas daquele país, poderiam ter contribuído para elevar o índice. Além disso, na janela de tempo entre o estudo canadense e o presente estudo, de três a seis anos, poderia ter ocorrido a incorporação de novas tecnologias de saúde com consequente aperfeiçoamento da qualidade da atenção à gestação e parto em Joinville, suficiente para prevenir as internações em UTI neonatal.

Em 2012, os quatro hospitais do município de Joinville possuíam aproximadamente quatro leitos de UTI neonatal para cada 1000 nascidos vivos, índice considerado adequado segundo o parâmetro recomendado pela Sociedade Brasileira de Pediatria. 23 Entretanto, considerando-se o total de nascidos vivos em 2012 nos hospitais públicos de Joinville (4568 nascidos vivos) e de outros sete municípios da $23^{\mathrm{a}}$ Região de Saúde do Estado cuja referência para partos de risco são os hospitais públicos de Joinville (2100 nascidos vivos), o total de leitos de UTI neonatal públicos cai para 2,5 leitos para cada mil nascidos vivos. Considerando que o Ministério da Saúde24 preconiza uma oferta de cinco leitos, dois de UTI e três de cuidados intermediários, evidencia-se o não atendimento a este parâmetro, caracterizando uma oferta insuficiente de atenção especializada aos recém-nascidos de risco do setor público nessa região. Como consequência, ocorre dificuldade no acesso das gestantes de risco que precisam ser referenciadas para maternidades localizadas em outros municípios, conforme os fluxos e pactuações definidos pelos colegiados regionais do SUS.

Em relação às variáveis maternas, identificou-se risco de internação em UTI neonatal 1,4 vezes maior para os recém-nascidos de mães sem companheiro e com baixa escolaridade quando comparados aos filhos de mães com companheiro e com maior escolaridade. Este resultado é similar a outros estudos nacionais onde se evidenciou piores resultados obstétricos entre mães com indicadores socioeconômicos desfavoráveis, especialmente a baixa escolaridade, considerada um marcador da condição socioeconômica da mãe e de sua família. 10,12,25

Os recém-nascidos de mães que tiveram menos de sete consultas de pré-natal tiveram um risco 1,3 vezes maior de internação do que aqueles cujas mães tiveram mais consultas. Este achado é similar ao de uma pesquisa realizada em Campinas (SP) que encontrou maior índice de recém-nascidos com baixo peso ao nascer entre as mães que tiveram menos de sete consultas no pré-natal. ${ }^{25}$ Em Pelotas (RS), Victora e Barros 10 chamam a atenção para o impacto potencial da melhoria da assistência prénatal na redução da mortalidade perinatal. Apesar do Ministério da Saúde recomendar ao menos sete consultas no pré-natal, 12 tem-se salientado atualmente a importância da qualidade da assistência ofertada na rede pública.

Observou-se predomínio de internação em UTI neonatal entre os nascidos vivos nos estabelecimentos públicos $(71 \%)$, tendo o risco de internação sido quase duas vezes maior entre os nascidos vivos nos hospitais públicos do que nos hospitais privados. Tal achado pode ser explicado em parte pelas características socioeconômicas das mães que frequentam os serviços públicos, tais como escolaridade, renda familiar e trabalho materno, que caracterizam um perfil de maior risco social, repercutindo negativamente na situação de saúde.25-27 Também, a dificuldade no acesso aos hospitais públicos por ocasião do parto e os problemas que afetam o acesso e a qualidade da assistência pré-natal na rede pública2,10,26 podem levar à perda de oportunidade para intervir adequadamente sobre os agravos durante a gestação. Tais fatores, de forma isolada ou associada, podem ocasionar ou agravar riscos dos recém-nascidos.

O presente estudo mostrou que a magnitude do efeito dos fatores de risco biológicos para a internação em UTI neonatal, como peso, malformações congênitas, Apgar de quinto minuto $<7$ e prematuridade, foi mais elevada quando comparada aos demais fatores investigados.

Os recém-nascidos com baixo peso ao nascer representaram $36,8 \%$ das internações neonatais com risco de internação cerca de oito vezes maior quando comparados aos recém-nascidos com peso normal. Verificou-se o mesmo com os recém-nascidos prematuros, que representaram $41,6 \%$ dos internados e um risco três vezes maior do que os recémnascidos de termo. Estes percentuais encontrados são inferiores aos de um estudo prospectivo realizado em maternidade pública na região nordeste que identificou $55,5 \%$ de prematuridade entre as crianças internadas em UTI neonatal 26 e de outro estudo transversal realizado em UTI neonatal de 
hospital de ensino na região sul do país, com índices de baixo peso de $39 \%$ e prematuridade de $66 \% .17$

$\mathrm{O}$ baixo peso é considerado o fator mais importante relacionado à morbimortalidade infantil.12,28 Os determinantes do baixo peso ao nascer incluem a prematuridade e a restrição de crescimento intrauterino, ou uma combinação de ambas. Dessa forma, os fatores envolvidos na gênese destas duas condições clínicas costumam sobrepor-se e aumentar a vulnerabilidade dos recém nascidos, em razão da instabilidade hemodinâmica e de suas propriedades fisiológicas. 5,7,12,14,16 Como consequência, eleva-se o risco de internação em UTI neonatal e de desfechos negativos, tais como complicações infecciosas e óbito. $2,5,7,14,17,26$

A prevalência da malformação congênita no presente estudo foi de 3,3\% nos recém nascidos internados e, apesar de sua ocorrência ter sido menor do que a encontrada em um estudo realizado em UTI neonatal de hospital público no sul do Brasil, que foi de 5,3\%,17 em nossa casuística a malformação congênita representou um risco seis vezes maior para internação em UTI neonatal. É possível que haja sub diagnóstico das malformações no presente estudo, uma vez que a fonte de dados utilizada foram as Declarações de Nascidos Vivos que são emitidas imediatamente após o parto, momento em que nem sempre é possível identificar-se certas malformações que só se manifestam clinicamente após algumas horas ou dias de vida. Considerada uma das principais causas básicas de óbitos perinatais em países desenvolvidos, 11 as malformações congênitas se destacam de forma crescente no Brasil, à medida que a mortalidade neonatal supera a pós-neonatal em magnitude. ${ }^{3}$ Estudo realizado em UTI neonatal de hospital público na Região Sul mostra que 25,6\% dos óbitos tiveram como causa básica as malformações congênitas. ${ }^{5}$ No Canadá, estudo realizado em 17 UTIs neonatais evidenciou 13,7\% dos recém nascidos internados com uma ou mais malformações congênitas, observando-se variação da incidência nas distintas regiões do país. ${ }^{11}$ Os autores chamam atenção não apenas para a associação entre malformações e maior morbimortalidade perinatal, mas para a elevada utilização de recursos ${ }^{11}$ nas unidades de UTI neonatais. Diante disso, o diagnóstico precoce na gestação pela ultrassonografia constitui fator primordial para a programação de intervenções oportunas. ${ }^{14}$ Atualmente, com a implantação da Rede Cegonha no Brasil, o Ministério da Saúde pretende universalizar a realização da ultrassonografia nos três trimestres de gestação. A ampliação da oferta deste exame na rede pública representa um grande desafio para qualificar a assistência pré-natal e ao parto. Porém, é preciso observar que também será necessário ofertar procedimentos especializados para os recém-nascidos diagnosticados com malformações, o que representa um desafio ainda maior no atual momento.

Neste estudo, 4,6\% dos recém-nascidos internados tiveram Apgar no quinto minuto menor que 7 , índice inferior ao encontrado em um estudo de coorte realizado em hospital público da cidade de Pelotas, entre 2008 e 2010, que foi de $12 \% .{ }^{7}$ Tal diferença em relação à nossa casuística poderia ser atribuída à melhor qualidade da assistência ao parto e ao recém-nascido na sala de parto dos hospitais de Joinville, conforme mostrado pela queda do índice de Apgar menor que 7 do primeiro minuto em relação ao quinto minuto de vida, observada entre todos os nascidos vivos e não apenas os que foram admitidos em UTI neonatal. Vale ressaltar que embora este escore isoladamente não seja suficiente para diagnosticar asfixia perinatal,29 ele possui relevância epidemiológica, já que os recém-nascidos com Apgar baixo possuem maior risco de internação em UTI e de óbito. 5 Neste estudo, o risco de internação em UTI neonatal foi quase seis vezes maior entre as crianças nascidas com Apgar no quinto minuto menor que 7 quando comparado com aquelas cujo Apgar foi maior que 7.

Recém-nascidos do sexo masculino apresentaram maior risco de internação quando comparados aos do sexo feminino. Em nosso estudo, 56,3\% dos recém nascidos eram do sexo masculino, índice similar aos de outros estudos que identificaram $52 \% 14$ e $57 \% .5,14,17$ A literatura aponta uma maior fragilidade pulmonar em recém nascidos do sexo masculino, decorrente de maturação mais lenta durante o crescimento fetal em comparação ao sexo feminino, 3 o que poderia explicar o predomínio de recém nascidos do sexo masculino internados um UTI.

É oportuno destacar que dentre os fatores associados ao risco de internação em UTI neonatal observados neste estudo estão dois critérios considerados na definição de near miss neonatal, como o Apgar de quinto minuto menor que 7 e presença de malformações congênitas, conforme apontou a pesquisa Nascer no Brasil, realizada em 2011 e 2012, que incluiu mais de 23 mil recém nascidos. ${ }^{29}$

Finalmente, é necessário contextualizar que os resultados do presente estudo podem não retratar a realidade de outras regiões do país, visto que o município analisado situa-se em uma das regiões mais desenvolvidas do Brasil com índices sociais, de desenvolvimento e de saúde acima da média nacional. Entretanto, ele produz uma referência 
nacional para se estimar a frequência de internações em UTI neonatal a partir de uma base populacional municipal, permitindo um planejamento da infraestrutura assistencial adequada para os recémnascidos de risco. As UTIs neonatais são serviços indispensáveis para melhorar a chance de sobrevivência dos recém-nascidos de alto risco, porém tanto os elevados custos das internações quanto os riscos de sequelas para os recém-nascidos, mostram que o investimento em prevenção ainda é a melhor opção. Nesse sentido, as características biológicas dos recém-nascidos, aqui evidenciadas como sendo

\section{Referências}

1. Richardson DK, Shah BL, Frantz III ID, Bednarek F, Rubin LP, McCormich MC. Perinatal risk and severity of illness in newborns at 6 Neonatal Intensive Care Units. Am J Public Health. 1999; 89 (4): 511-6.

2. Weirich CF, Domingues MHMS. Mortalidade neonatal um desafio para os serviços de Saúde. Rev Eletrônica Enferm. 2001; 3 (1). [acesso em 4 dez 2014]. Disponível em http://www.revistas.ufg.br/index.php/fen/article/view/696/7 73

3. Ribeiro AM, Guimarães MJ, Lima MC, Sarinho SW, Coutinho SB. Fatores de risco para mortalidade neonatal em crianças com baixo peso ao nascer. Rev Saúde Pública. 2009; 43 (2): 246-55

4. Brasil. Ministério da Saúde. Secretaria de Vigilância em Saúde. Secretaria de Atenção à Saúde. Manual de vigilância do óbito infantil e fetal e do comitê de prevenção do óbito infantil e fetal. Normas e Manuais Técnicos. Brasília, DF; 2009

5. Araújo BFA, Tanaka ACA, Madi JM, Zatti H. Estudo da mortalidade de recém-nascidos internados na UTI neonatal do Hospital Geral de Caxias do Sul, Rio Grande do Sul. Rev Bras Saúde Matern Infant. 2005; 5 (4): 463-9.

6. Gonçalves AC, Costa, MCN, Braga JU. Análise da distribuição espacial da mortalidade neonatal e de fatores associados, em Salvador, Bahia, Brasil, no período 20002006. Cad Saúde Pública. 2011; 27 (8): 1581-92.

7. Granzotto JA, Mota DM, Real RF, Dias CM, Teixeira RF, Menta Filho JC, Tiecher GB, Pilecco AJL, Gonçalves ER. Análise do perfil epidemiológico das internações em uma Unidade de Terapia Intensiva Neonatal. Rev AMRIGS. 2012; 56 (4): 304-7.

8. Ribeiro ERRO, Barbieri MA, Bettiol H, Silva AAM Comparação entre duas coortes de mães adolescentes em município do Sudeste do Brasil. Rev Saúde Pública. 2000; 34 (2): 136-42.

9. Shrim A, Ales S, Mallozi A, Brown R, Ponette V, Levin I, Shehata F, Almog B. Is young maternal age really a risk factor for adverse pregnancy outcome in a Canadian tertiary referral hospital? J Pediatr Adolesc Gynecol. 2011; 24 (4): 218-22.

10. Victora CG, Barros FC. Infant mortality due to perinata causes in Brazil: trends, regional patterns and possible interventions. Rev Paul Med 2001; 119 (1): 33-42. os riscos mais significativos para internação em UTI neonatal são, em geral, passíveis de prevenção e sugerem que o direcionamento dos recursos no âmbito de saúde pública deve se dar na busca da melhoria da qualidade da assistência à gestante e ao recém-nascido.

\section{Agradecimentos}

À Prof. Dra. Elisa Henning da Universidade do Estado de Santa Catarina (UDESC), que colaborou nas análises estatísticas deste trabalho.

11. Synnes AR, Berry M, Jones H, Pendray M, Stewart S, Lee SK. Infants with congenital anomalies admitted to neonatal intensive care units. Am J Perinatol. 2004; 21 (4): 199-207.

12. Brasil. Ministério da Saúde. Secretaria de Atenção à Saúde. Departamento de Ações Programáticas e Estratégicas. Atenção à saúde do recém-nascido: guia para os profissionais de saúde 4, série A. Normas e Manuais Técnicos. Brasília, DF; 2011.

13. Battin M, Sadler L. Neonatal intensive care utilization and neonatal outcome of infants born to women aged 40 years and over in New Zealand. Acta Pediatr. 2010; 99 (2): 219 24.

14. Basso CG, Neves ET, Silveira A. The association between attending prenatal care and neonatal morbidity. Texto Contexto Enferm. 2012; 21 (2): 269-76.

15. Greenwood S, Abdel-Latif ME, Bajuk B, Lui K. Can the early condition at admission of a high-risk infant aid in the prediction of mortality and poor neurodevelopmental outcome? A population study in Australia. J Paediatr Child Health. 2012; 48 (7): 588-95.

16. WHO (World Health Organization). Save the Children, WHO Born Too Soon: The Global Action Report on Preterm Birth. Eds CP Howson, MV Kinney, JE Lawn. 2012; 2005 [acesso em 12 mai 2014]. Disponível em http://www.who.int/pmnch/media/news/2012/201204_born toosoon-report.pdf

17. Tadielo BZ, Neves ET, Arrué AM, Silveira A, Ribeiro AC, Tronco CS, Neves AT, Weis PSC. Morbidade e Mortalidade de recém-nascidos em tratamento intensivo neonatal no sul do Brasil. Rev Soc Bras Enferm Ped. 2013; 13 (1): 7-12.

18. Turner D, Simpson P, Li SH, Scanlon M, Quasney MW. Racial disparities in pediatric intensive care unit admissions. South Med J. 2011; 104 (9): 640-6.

19. Carter MF, Xenakis E, Holden A, Dudley D. Neonatal intensive care unit admissions and their associations with late preterm birth and maternal risk factors in a populationbased study. J Matern Fetal Neonatal Med. 2012; 25 (4): 343-5.

20. Chelliah AM, Vilchez G, Dai J, Bahado-Singh RO, Sokol RJ. Risk factors for neonatal intensive care unit admission after term twin deliveries. Obstet Gynecol. 2014; 123 (Supl. 1): $141 \mathrm{~S}$ 
21. Jorge MHPM, Gotlieb SLD, Soboll, MLMS, Almeida MF, Latorre MRDO. Avaliação do Sistema de Informação sobre Nascidos Vivos e o Uso de seus Dados em Epidemiologia e Estatísticas de Saúde. Rev Saúde Pública. 1993; 27 (Supl. 6): $1-46$

22. Fallah S, Chen XK, Lefebvre D, Kurji J, Hader J, Leeb K. Babies admitted to NICU/ICU: province of birth and mode of delivery matter. Healthc Q. 2011; 14 (2): 16-20.

23. Fiori RM. Regionalização da assistência perinatal. In: Miranda LEV, Lopes JMA, organizadores. Manual de Perinatologia. Rio de Janeiro: Sociedade Brasileira de Pediatria; 1991. p. 17-20

24. Brasil. Ministério da Saúde. Portaria ${ }^{\circ}$ 930, de 10 de maio de 2012. [acesso em 18 jun 2014]. Define as diretrizes e objetivos para a organização da atenção integral e humanizada ao recém-nascido grave ou potencialmente grave e os critérios de classificação e habilitação de leitos de Unidade Neonatal no âmbito do Sistema Único de Saúde (SUS). Disponível em: http://bvsms.saude.gov.br/ bvs/saudelegis/gm/2012/prt0930_10_05_2012.html
25. Carniel EF, Zanolli M, Antonio MARGM, Morcillo AM. Determinantes do baixo peso ao nascer a partir das Declarações de Nascidos Vivos, 2008. Rev Bras Epidemiol. 2008; 1 (11): 169-79.

26. Costa ALRR, Araujo Junior E, Lima JWO, Costa FS. Fatores de risco materno associados à necessidade de unidade de terapia intensiva neonatal. Rev Bras Ginecol Obstet. 2014; 36 (1): 29-34

27. Mandarino NR, Chein MBC, Monteiro Jr FC, Brito LMO, Lamy ZC, Nina VJS, Mochel EG, Figueiredo Neto JA. Aspectos relacionados à escolha do tipo de parto: um estudo comparativo entre uma maternidade pública e outra privada, em São Luís, Maranhão, Brasil. Cad Saúde Pública. 2009; 25 (7): 1587-96.

28. Kramer MS. Determinants of low birth weight: methodological assessment and meta-analysis. Bull World Health Organ. 1987; 65 (5): 663-737.

29. Silva AAM, Leite AJM, Lamy ZC, Moreira MEL, Gurgel RQ, Cunha AJLA, Leal MC. Morbidade neonatal near miss na pesquisa Nascer no Brasil. Cad Saúde Pública. 2014; 30 (Supl.): S182-91.

Recebido em 17 de dezembro de 2014

Versão final apresentada em 10 de março de 2015

Aprovado em 1 de abril de 2015 